\title{
MVDR BROADBAND BEAMFORMING USING POLYNOMIAL MATRIX TECHNIQUES
}

\author{
S. Weiss ${ }^{1}$, S. Bendoukha ${ }^{2}$, A. Alzin ${ }^{1}$, F.K. Coutts ${ }^{1}$, I.K. Proudler ${ }^{3}$, J. Chambers ${ }^{3,4}$ \\ ${ }^{1}$ Department of Electronic \& Electrical Engineering, University of Strathclyde, Glasgow, Scotland \\ ${ }^{2}$ Department of Electrical Engineering, College of Engineering, Taibah University, Yanbu, Saudi Arabia \\ ${ }^{3}$ School of Electrical, Electronics \& Systems Engineering, Loughborough Univ., Loughborough, UK \\ ${ }^{4}$ School of Electrical and Electronic Engineering, Newcastle University, Newcastle upon Tyne, UK
}

\begin{abstract}
This paper presents initial progress on formulating minimum variance distortionless response (MVDR) broadband beamforming using a generalised sidelobe canceller (GSC) in the context of polynomial matrix techniques. The quiescent vector is defined as a broadband steering vector, and we propose a blocking matrix design obtained by paraunitary matrix completion. The polynomial approach decouples the spatial and temporal orders of the filters in the blocking matrix, and decouples the adaptive filter order from the construction of the blocking matrix. For off-broadside constraints the polynomial approach is simple, and more accurate and considerably less costly than a standard time domain broadband GSC.
\end{abstract}

\section{INTRODUCTION}

If broadband signals need to be resolved by an array, sensor elements usually have to be followed by tap delay lines in order to capture the relative lag rather than just a phase shift between signals. This has led to the extension of many narrowband beamforming techniques to the broadband case, such as minimum variance distortionless response and one of its realisations, the generalised sidelobe canceller [1].

To describe broadband array signals and MIMO systems, covariance and transfer functions can be denoted as polynomial matrices [2]. For such quantities, narrowband techniques such as EVD and SVD have been extended to polynomials, with applications in denoising-type [3] or decorrelating preprocessors [4], transmit and receive beamforming across broadband MIMO channels [5,6], broadband angle of arrival estimation [7], and for optimum subband partitioning of beamformers [8].

Here, polynomial matrix techniques are extended to the MVDR problem. We will demonstrate below that this can provide an elegant framework particulary when designing beamformers with arbitrary look direction. Most beamformer designs assume presteering and look towards broad-

This work was supported in parts by the Engineering and Physical Sciences Research Council (EPSRC) Grant number EP/K014307/1 and the MOD University Defence Research Collaboration in Signal Processing. side [9-11], where the constraint can be inexpensively implemented [12]. This work complements the sparse literature on designs with an arbitary look direction [13], with a significant reduction in complexity.

\section{MVDR BEAMFORMING}

This section briefly reviews narrowband MVDR beamforming in Sec. 2.1 and comments on its standard extension to the broadband case in Sec. 2.2.

\subsection{MVDR and Generalised Sidelobe Canceller}

If $\mathbf{x}[n] \in \mathbb{C}^{M}$ is a vector of sensor signals with space-time covariance $\mathbf{R}[\tau]=\mathcal{E}\left\{\mathbf{x}[n] \mathbf{x}^{\mathrm{H}}[n-\tau]\right\}$, and $e[n]=\mathbf{w}^{\mathrm{H}} \mathbf{x}[n]$ the output of a beamformer with coefficients in $\mathbf{w}$, then the narrowband MVDR beamformer optimises the constrained problem

$$
\begin{array}{ll} 
& \min _{\mathbf{w}} \mathcal{E}\left\{|e[n]|^{2}\right\}=\min _{\mathbf{w}} \mathbf{w}^{\mathrm{H}} \mathbf{R}[0] \mathbf{w} \\
\text { s.t. } & \mathbf{s}^{\mathrm{H}}\left(\vartheta_{\mathrm{s}}, \Omega\right) \mathbf{w}=1
\end{array}
$$

whereby the constraint by a steering vector $\mathbf{s}^{\mathrm{H}}\left(\vartheta_{\mathrm{s}}, \Omega\right)$ protects the signal of interest in look direction $\vartheta_{\mathrm{s}}$ at normalised angular frequency $\Omega$.

Instead of solving the MVDR problem directly, a GSC forms a quiescent beamformer $\mathrm{w}_{\mathrm{q}}$ which points in direction $\vartheta_{\mathrm{s}}$ and minimises (1) for spatially white noise. Its output $d[n]=\mathbf{w}_{\mathrm{q}}^{\mathrm{H}} \mathbf{x}[n]$ in general still contains interference. Therefore, a blocking matrix $\mathbf{B}$, whose rows span the nullspace of $\mathbf{w}_{\mathrm{q}}$ such that $\mathbf{B} \mathbf{w}_{\mathrm{q}}=\underline{0}$, can generate a signal $\mathbf{u}[n]=\mathbf{B} \mathbf{x}[n]$ containing interference only. In an unconstrained optimisation step, a noise cancelling filter $\mathbf{w}_{\mathrm{a}}$ can therefore be applied such that $e[n]=d[n]-\mathbf{w}_{\mathrm{a}}^{\mathrm{H}} \mathbf{u}[n]$ contains no more interference, enforcing $\mathcal{E}\left\{\mathbf{u}[n] e^{*}[n]\right\}=\underline{0}$.

\subsection{Broadband Case}

A broadband MVDR or GSC incorporates tap delay lines following each sensor, and in standard notation all quantities in Sec. 2.1 are expanded by a temporal dimension [1], $L$, such 


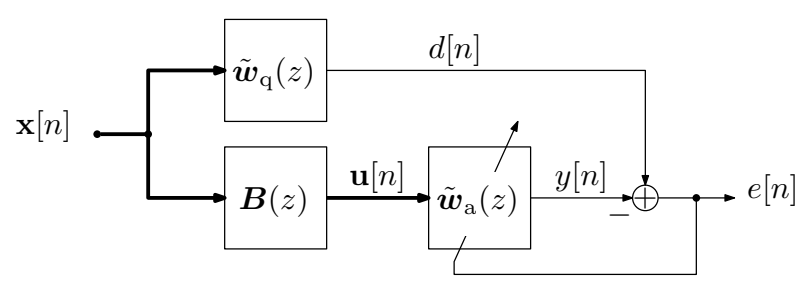

Fig. 1. Generalised sidelobe canceller with polynomial quiescent vector and polynomial blocking matrix; the system $\boldsymbol{w}_{\mathrm{a}}(z)$ represents a multichannel adaptive filter.

that e.g. $\mathbf{x} \in \mathbb{C}^{M L}$. While this is generally straightforward, we here focus on the formulation of constraints. A constraint matrix $\mathbf{C}$ contains columns of steering vectors in direction of $\vartheta_{\mathrm{s}}$ over a set of frequencies,

$$
\mathbf{C}=\left[\mathbf{s}\left(\vartheta_{\mathrm{s}}, \Omega_{0}\right), \mathbf{s}\left(\vartheta_{\mathrm{s}}, \Omega_{1}\right) \ldots \mathbf{s}\left(\vartheta_{\mathrm{s}}, \Omega_{L-1}\right)\right]
$$

and the constraint equation $\mathbf{C}^{\mathrm{H}} \mathbf{W}=\mathbf{f}$ defines the desired frequency response in $\mathbf{f} \in \mathbb{C}^{L}$ for look direction $\vartheta_{\mathrm{s}}$. For a planar array, the simplest constraint is towards broadside, where $\mathbf{C}$ is assembled from $L$ identity matrices, but in principle, the $L$ point constraints defined via (3) can also be applied for an off-broadside look direction [13].

\section{PROPOSED APPROACH}

\subsection{Polynomial MVDR and GSC}

Defining $\boldsymbol{w}(z) \in \mathbb{C}^{M}$ to contain the complex conjugated and time-reversed $M$ beamforming filters and $\boldsymbol{R}(z) \bullet \longrightarrow \mathbf{R}[\tau]$ as the $z$-transform of space-time covariance matrix, the output power spectral density of the beamformer is $R_{e}(z)=$ $\tilde{\boldsymbol{w}}(z) \boldsymbol{R}(z) \boldsymbol{w}(z)$ leads to the MVDR problem formulation

$$
\begin{array}{ll} 
& \min _{\mathbf{w}(z)} \oint_{|z|=1} R_{e}(z) \frac{d z}{z} \\
\text { s.t. } & \tilde{\boldsymbol{s}}\left(\vartheta_{\mathrm{s}}, z\right) \boldsymbol{w}(z)=F(z),
\end{array}
$$

with the parahermitian transpose operation $\tilde{\boldsymbol{w}}(z)=\boldsymbol{w}^{\mathrm{H}}\left(z^{-1}\right)$. The broadband steering vector $s\left(\vartheta_{\mathrm{s}}, z\right)$ defines the look direction of the array with a desired frequency response $F(z)$. For simplicity $F(z)=1$ is assumed.

To solve (4) and (5), we propose the polynomial GSC shown in Fig. 1. The quiescent beamformer $\mathbf{w}_{\mathrm{q}}(z)$ is derived from the constraint (5), which in turn defines a blocking matrix $\boldsymbol{B}(z)$ and a multichannel adaptive filter with coefficients in $\boldsymbol{w}_{\mathrm{a}}(z)$. Below, these components are elaborated in turn.

\subsection{Broadband Steering Vector and Quiescent Beam- former}

A broadband steering vector $s(\vartheta, z)$ contains explicit delays rather than phase shifts as in the narrowband case, such that

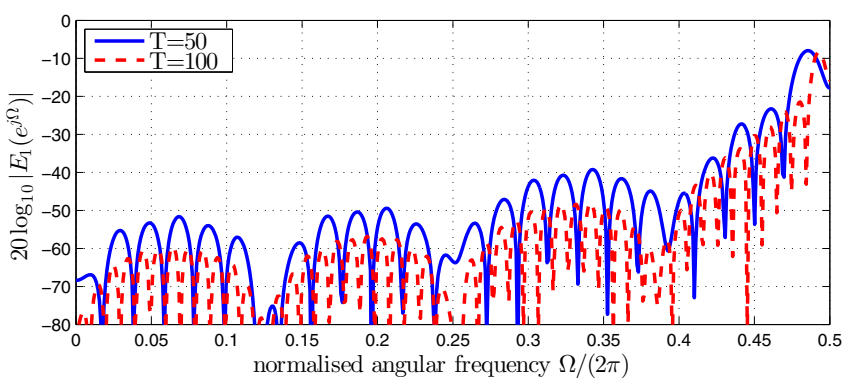

Fig. 2. Error $E_{2}(z)=\tilde{\boldsymbol{s}}\left(30^{\circ}, z\right) \boldsymbol{s}\left(30^{\circ}, z\right)-1$ evaluated on the unit circle, with windowed sinc functions of order $T$ as fractional delay filters.

$\mathbf{s}_{\vartheta}[n] \circ \longrightarrow \boldsymbol{s}(\vartheta, z)$

$$
\mathbf{s}_{\vartheta}[n]=\frac{1}{\sqrt{M}}\left[\begin{array}{c}
d[n] \\
d\left[n-\tau_{2}(\vartheta)\right] \\
\vdots \\
d\left[n-\tau_{M}(\vartheta)\right]
\end{array}\right],
$$

where $d[n-\tau]$ is an ideal fractional delay by $\tau \in \mathbb{R}$ samples. A waveform from direction $\vartheta$ experiences a lag $\tau_{m}(\vartheta)$ relative to element $m=1$ when it arrives at the $m$ th sensor. Evaluating (6) at frequency $\Omega$ turns the delays $d\left[n-\tau_{m}(\vartheta)\right]$ into phase shifts and $\mathbf{s}_{\vartheta}[n]$ into a narrowband steering vector as discussed in Sec. 2.1. With (6), $\tilde{\boldsymbol{s}}(\vartheta, z) \boldsymbol{s}(\vartheta, z)=1$ is easily verified.

To implement a broadband steering vector according to (6) requires fractional delay filters. With relatively moderate order, high accuracy can be achieved close up to half of the sampling rate using e.g. windowed sinc functions $[14,15]$. An example for the accuracy of $s(\vartheta, z)$ with $\vartheta=30^{\circ}$ for a linear, critically sampled array with $M=8$ equispaced elements is shown in Fig. 2.

Assuming that $\tilde{\boldsymbol{s}}(\vartheta, z) \boldsymbol{s}(\vartheta, z) \approx 1$ for the steering vectors constructed above, for $F(z)=1(5)$ is fulfilled with $\boldsymbol{w}_{\mathrm{q}}(z)=$ $\boldsymbol{s}\left(\vartheta_{\mathrm{s}}, z\right)$. Therefore, the output of the quiescent beamformer is

$$
d[n]=\sum_{\nu=0}^{T} \mathbf{w}_{\mathrm{q}}^{\mathrm{H}}[-\nu] \mathbf{x}[n-\nu] .
$$

Note that $\boldsymbol{w}_{\mathrm{q}}(z) \bullet \mathrm{w}_{\mathrm{q}}[n]$ is of order $T$ and holds the parahermitian transpose of the actual coefficients.

\subsection{Blocking Matrix}

The blocking matrix has to be designed such that

$$
\boldsymbol{B}(z) \boldsymbol{w}_{\mathrm{q}}(z)=\underline{0}
$$

To achieve orthonormality between $\boldsymbol{B}(z)$ and $\boldsymbol{w}_{\mathrm{q}}(z)$, a paraunitary matrix

$$
\boldsymbol{Q}(z)=\left[\mathbf{w}_{\mathrm{q}}(z) \tilde{\boldsymbol{B}}(z)\right]
$$


can be constructed with $\boldsymbol{Q}(z) \tilde{\boldsymbol{Q}}(z)=\mathbf{I}$ and $\tilde{\boldsymbol{B}}(z)=$ $\boldsymbol{B}^{\mathrm{H}}\left(z^{-1}\right)$. For the narrowband or standard broadband cases using matrices and vectors with scalar entries, this can be achieved by a variety of methods such as singular value decomposition of the constraint vector/matrix, or orthogonalisation of the columns in (9) using Gram-Schmidt or QR decompositions [16]. However, the polynomial case is more involved and will be separately addressed in Sec. 4.

The output of the blocking matrix, $\mathbf{u}[n] \in \mathbb{C}^{M-1}$ as shown in Fig. 1, is

$$
\mathbf{u}[n]=\sum_{\nu=0}^{N} \mathbf{B}[\nu] \mathbf{x}[n-\nu],
$$

where $\mathbf{B}[n] \circ \longrightarrow \boldsymbol{B}(z)$ is of order $N$. This order $N$ impacts on the computational complexity of $\boldsymbol{B}(z)$ and will arise from its construction in Sec. 4.

\subsection{Multichannel Noise Cancellation}

With $\boldsymbol{w}_{\mathrm{q}}(z)$ and $\boldsymbol{B}(z)$ as defined previously, a multichannel filter $\boldsymbol{w}_{\mathrm{a}}(z) \in \mathbb{C}^{M-1}$ can be employed to remove the remaining interference from the quiescent beamformer output $d[n]$ using $\mathbf{u}[n]$, as shown in Fig. 1 . With $\boldsymbol{w}_{\mathrm{a}}(z)$ containing the parahermitian of the actual filter coefficients, the beamformer output is

$$
e[n]=d[n]-\sum_{\nu=0}^{L} \mathbf{w}_{\mathrm{a}}^{\mathrm{H}}[-\nu] \mathbf{u}[n-\nu],
$$

whereby $\mathbf{w}_{\mathrm{a}}[n] \curvearrowright \boldsymbol{w}_{\mathrm{a}}(z)$ is of order $L$.

The multichannel filter $\boldsymbol{w}_{\mathrm{a}}(z)$ can be determined through unconstrained minimisation of $\mathcal{E}\left\{|e[n]|^{2}\right\}$. Various tools exist, such as MMSE or Wiener solution, as well as adaptive techniques such as LMS or RLS [17]. For simulations in Sec. 6, the multichannel normalised LMS (NLMS) algorithm will be used.

\section{PARAUNITARY MATRIX COMPLETION}

This section proposes a paraunitary matrix completion to find a $\boldsymbol{Q}(z)$ in (9) based on $\boldsymbol{w}_{\mathrm{q}}(z)$. For this, we employ a polynomial eigenvalue decomposition (PEVD, [18]) of the rank one matrix

$$
\boldsymbol{w}_{\mathrm{q}}(z) \tilde{\boldsymbol{w}}_{\mathrm{q}}(z)=\overline{\boldsymbol{Q}}(z) \boldsymbol{D}(z) \tilde{\overline{\boldsymbol{Q}}}(z) .
$$

The PEVD approximately diagonalises and spectrally majorises $\boldsymbol{D}(z)$ by means of a paraunitary matrix $\overline{\boldsymbol{Q}}(z)$. Spectral majorisation is equivalent to ordering in the SVD [16], and ensures that the energy is compacted into as few polynomial eigenvalues in $\boldsymbol{D}(z)$ as possible. Since $\boldsymbol{w}_{\mathrm{q}}(z)$ has unit norm and (12) is rank one by construction, we obtain

$$
\boldsymbol{D}(z)=\operatorname{diag}\left\{\begin{array}{llll}
1 & 0 & \ldots & 0
\end{array}\right\} .
$$

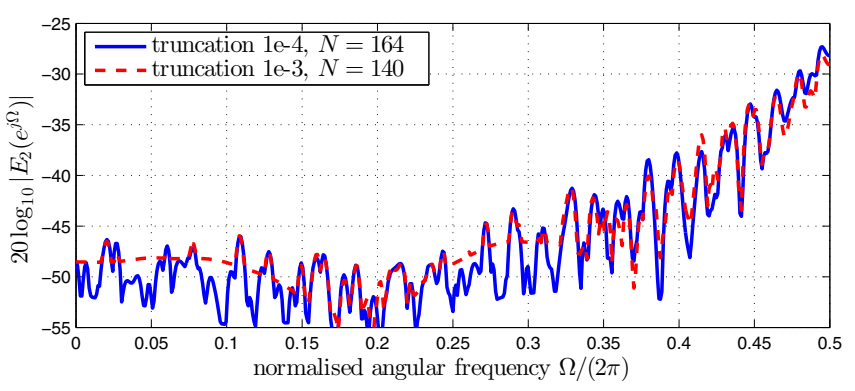

Fig. 3. Leakage of blocking matrix according to (17).

The paraunitary matrix $\overline{\boldsymbol{Q}}(z)$ is ambiguous even if (12) had full rank. If

$$
\overline{\boldsymbol{Q}}(z)=\left[\begin{array}{llll}
\overline{\boldsymbol{q}}_{1}(z) & \overline{\boldsymbol{q}}_{2}(z) & \ldots & \overline{\boldsymbol{q}}_{M}(z)
\end{array}\right]
$$

then $\overline{\boldsymbol{q}}_{1}(z)$ could e.g. be a shifted version of the polynomial vectors $\boldsymbol{w}_{\mathrm{q}}(z)$,

$$
\overline{\boldsymbol{q}}_{1}(z)=z^{-\Delta} \boldsymbol{w}_{\mathrm{q}}(z),
$$

and still satisfy both (12) and (13). Similarly, the remaining columns $\overline{\boldsymbol{q}}_{m}(z)$ could be arbitrarily shifted. Therefore, when defining

$$
\tilde{\boldsymbol{B}}(z)=\left[\begin{array}{lll}
\overline{\boldsymbol{q}}_{2}(z) & \ldots & \overline{\boldsymbol{q}}_{M}(z)
\end{array}\right],
$$

$\tilde{\boldsymbol{B}}(z) \boldsymbol{w}_{\mathrm{q}}(z)=\underline{0}$ is guaranteed, but $\boldsymbol{B}(z)$ may have a larger order than necessary. Through appropriate shift of rows and truncation of small outer coefficients of $\boldsymbol{B}(z)$ [19], this order can be reduced.

Example. Using the previous example of $\boldsymbol{w}_{\mathrm{q}}(z)=$ $\boldsymbol{s}\left(30^{\circ}, z\right)$ with $T=50$ and the above procedure, $\boldsymbol{B}(z)$ is calculated by sequential matrix diagonalisation [20], which implements an iterative PEVD algorithm. To measure how much of the signal of interest leaks through the blocking matrix - which can result in signal cancellation in the GSC the following error metric defined over a set of frequencies $\left\{\Omega_{i}\right\}$,

$$
E_{2}\left(e^{j \Omega_{i}}\right)=\max _{m \in\{2 \ldots M\}}\left|\overline{\boldsymbol{q}}_{m}^{\mathrm{H}}\left(e^{-j \Omega_{i}}\right) \boldsymbol{w}_{\mathrm{q}}\left(e^{j \Omega_{i}}\right)\right|
$$

extracts the maximum error across all $M-1$ inner products at every frequency. The result for truncation of $\boldsymbol{B}(z)$ by $1 \%$ and $0.1 \%$ of its energy is shown in Fig. 3. The error is acceptable particularly at low frequencies. It is dominated by inaccuracies in the construction of the broadband steering vector, but not by the iterative PEVD or the truncation of $\boldsymbol{B}(z)$.

\section{PERFORMANCE METRICS}

Directivity Pattern. The directivity pattern measures the gain response of a broadband beamformer with respect to AoA and frequency. With a broadband source at angle $\vartheta$ characterised 
Table 1. Computational complexity of different broadband beamformer realisations in multiply accumulates (MACs).

\begin{tabular}{|l|c|c|}
\hline \multirow{2}{*}{ component } & \multicolumn{2}{|c|}{ GSC cost } \\
\cline { 2 - 3 } & polynomial & standard \\
\hline \hline quiescent beamformer & $M(T+1)$ & $M(L+1)$ \\
\hline blocking matrix & $M(M-1)(N+1)$ & $M(M-1)(L+1)^{2}$ \\
\hline adaptive filter (NLMS) & $2(M-1)(L+1)$ & $2(M-1)(L+1)$ \\
\hline
\end{tabular}

by the broadband steering vector $s(\vartheta, z)$, the overall transfer function of the source and beamformer is

$$
A(\vartheta, z)=\left(\tilde{\boldsymbol{w}}_{\mathrm{q}}(z)-\tilde{\mathbf{w}}_{\mathrm{a}}(z) \boldsymbol{B}(z)\right) \cdot \boldsymbol{s}(\vartheta, z) .
$$

The directivity pattern is the magnitude of the response $A\left(\vartheta, e^{j \Omega}\right)$, which is obtained by probing (18) with a series of steering vectors and evaluating it on the unit circle.

Residual Error. To assess convergence of the optimisation methods for $\boldsymbol{w}_{\mathrm{a}}(z)$, a useful metric is to assess the mean square of the residual error $e_{\mathrm{r}}[n]$, obtained by subtracting the source signal projected through the quiescent vector from the error $e[n]$.

Computational Cost. The computational complexity of the various polynomial GSC components in Sec. 3 is listed in Table 1. For comparison, the costs for a time domain broadband beamformer is also stated [12]. An off-broadside look direction can be enforced through point constraints in the frequency domain, but prevents simplifications to the blocking matrix, which has to be applied to the full spatio-temporal data vector of dimension $M L$.

\section{SIMULATIONS AND RESULTS}

We assume a signal of interest from $\vartheta=30^{\circ}$, and three interferers from angles $\vartheta_{i} \in\left\{-40^{\circ},-10^{\circ}, 80^{\circ}\right\}$ active over the frequency range $\Omega=2 \pi \cdot[0.1 ; 0.45]$ at signal to interference ratio of $-40 \mathrm{~dB}$. The $M=8$ element linear uniform array is also corrupted by spatially and temporally white additive Gaussian noise at $20 \mathrm{~dB}$ SNR.

An example for the directivity pattern of $\boldsymbol{w}_{\mathrm{q}}(z)=$ $s\left(30^{\circ}, z\right)$ with $T=50$, is shown in Fig. 4. A time domain broadband quiescent beamformer designed from $T+1$ point constraints in the frequency domain is provided as a benchmark in Fig. 5. Both beamformers are similar, but while the polynomial version has inaccuracies in look direction towards $\Omega=\pi$ due to the broadband steering vectors lacking precision, the standard approach has inaccuracies particularly at the lower end of the spectrum, as will be seen later in Fig. 9.

With a quiescent design of $T=50$ as shown previously and a blocking matrix via PEVD completion with order $N=$ 140 , an $L=175$ order NLMS algorithm optimises $\boldsymbol{w}_{\mathrm{a}}(z)$. The convergence curve is shown in Fig. 6, together with that of a standard time domain broadband GSC of same dimension $L$. The directivity patterns with converged $\boldsymbol{w}_{\mathrm{a}}(z)$ are

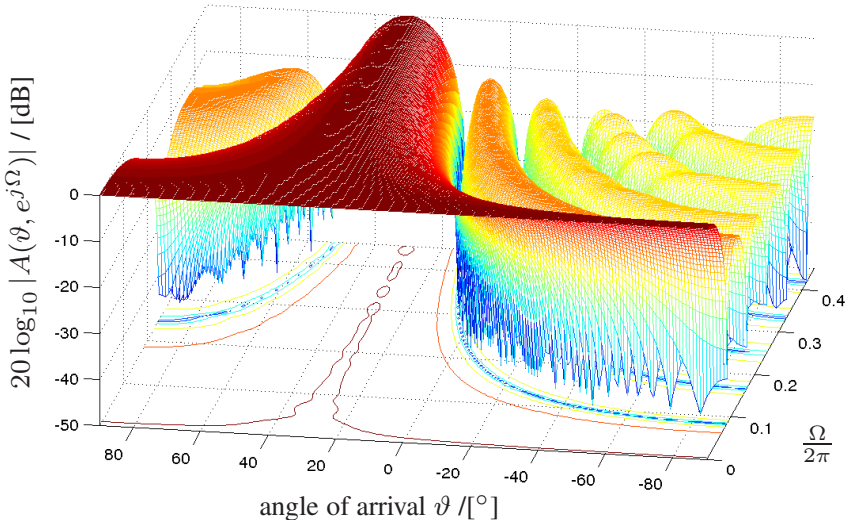

Fig. 4. Directivity pattern of polynomial quiescent beamformer with look direction $\vartheta_{\mathrm{s}}=30^{\circ}$.

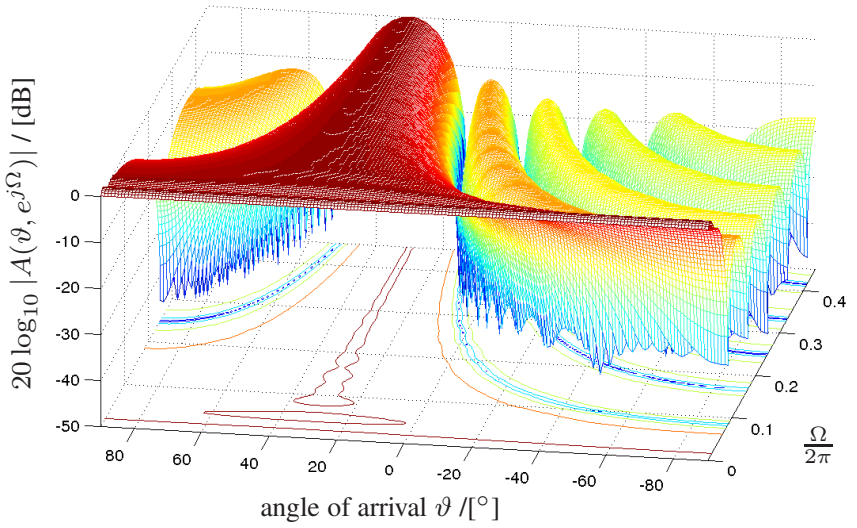

Fig. 5. Directivity pattern of standard broadband quiescent beamformer with look direction $\vartheta_{\mathrm{s}}=30^{\circ}$.

in Fig. 7 for the proposed polynomial approach and Fig. 8 for the benchmark. Both beamformers have placed nulls towards the three interferers, but the polynomial approach protects the constraint better - an example for the gain in look direction, which is constrained to $0 \mathrm{~dB}$, is shown in Fig. 9 for $T=L=50$ before and after adaptation. While the standard approach oscillates strongly between its point constraints, the polynomial approach is much better behaved.

With the above parameters and the cost as listed in Table 1 , the proposed beamformer requires $10.7 \mathrm{kMACs}$, while the standard broadband beamformer takes almost $L$ times as much with 1.72 MMACs per iteration step.

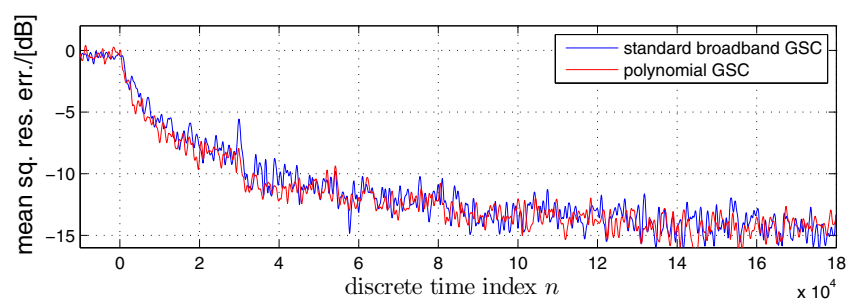

Fig. 6. Mean square residual error for proposed polynomial GSC and standard time domain GSC using the NLMS. 


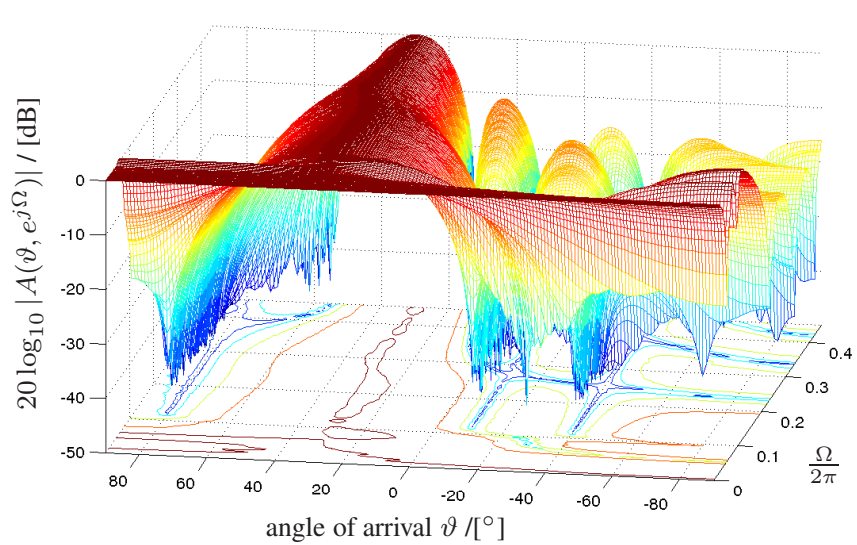

Fig. 7. Directivity pattern of adapted polynomial GSC.

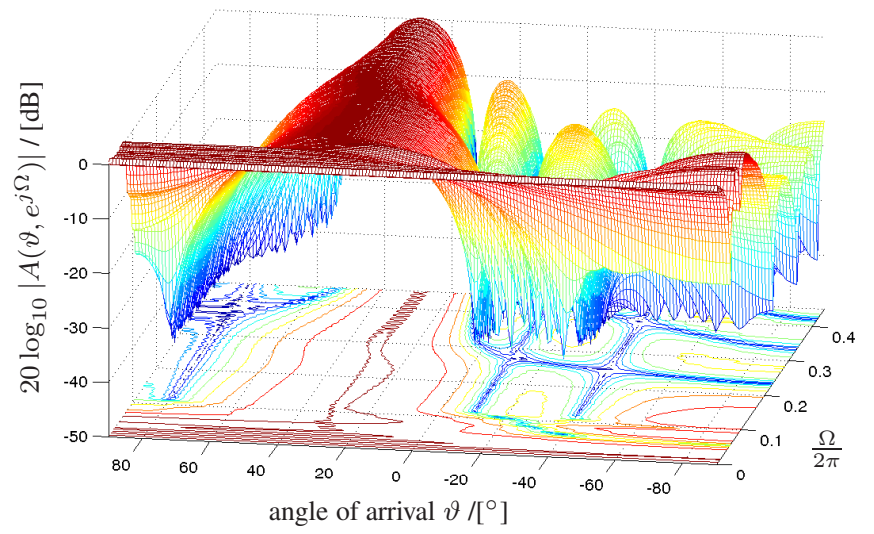

Fig. 8. Directivity pattern of adapted standard GSC.

\section{CONCLUSIONS}

A polynomial matrix formulation of a GSC implementing an MVDR beamformer has been introduced, which requires the definition of constraints via broadband steering vectors. For the construction of the blocking matrix, a paraunitary matrix completion has been defined. The proposed method can elegantly and compactly handle off-broadside constraints and define metrics such as the directivity pattern, and can lead to accurate beamformers of considerably lower complexity compared to the standard time domain counterpart.

\section{REFERENCES}

[1] B.D. Van Veen and K.M. Buckley, "Beamforming: a versatile approach to spatial filtering," IEEE ASSP Mag., 5(2):4-24, Apr. 1988.

[2] P.P. Vaidyanathan, Multirate systems and filter banks, Prentice Hall, 1993.

[3] S. Redif, J.G. McWhirter, P.D. Baxter, and T. Cooper, "Robust broadband adaptive beamforming via polynomial eigenvalues," in OCEANS, Boston, MA, Sep. 2006, pp. 1-6.

[4] C.L. Koh, S. Redif, and S. Weiss, "Broadband GSC beamformer with spatial and temporal decorrelation," in $E U$ SIPCO, Glasgow, UK, Aug. 2009, pp. 889-893.

[5] M. Davies, S. Lambotharan, and J.G. McWhirter, "Broadband MIMO beamforming using spatial-temporal filters and polynomial matrix decomposition," in Int. Conf. DSP, Cardiff, UK, July 2007, pp. 579-582.

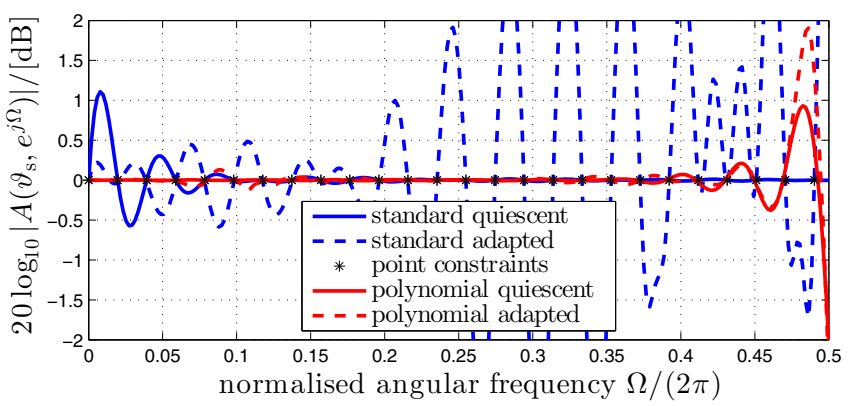

Fig. 9. Gain in look direction $\vartheta_{\mathrm{s}}=30^{\circ}$ before and after adaptation.

[6] C.H. Ta and S. Weiss, "A design of precoding and equalisation for broadband MIMO systems," in Int Conf. DSP, Cardiff, UK, July 2007, pp. 571-574.

[7] M. Alrmah, S. Weiss, and S. Lambotharan, "An extension of the MUSIC algorithm to broadband scenarios using polynomial eigenvalue decomposition," in EUSIPCO, Barcelona, Spain, Aug. 2011, pp. 629-633.

[8] P.G. Vouras and T.D. Tran, "Robust transmit nulling in wideband arrays," IEEE Trans. SP, 62(14):3706-3719, July 2014.

[9] I. Thng, A. Cantoni, and Y.H. Leung, "Derivative constrained optimum broad-band antenna arrays," IEEE Trans. SP, 41(7):2376-2388, July 1993.

[10] S. Zhang and I.L.-J. Thng, "Robust presteering derivative constraints for broadband antenna arrays," IEEE Trans. SP, 50(1):1-10, Jan. 2002.

[11] M. Rübsamen and A.B. Gershman, "Robust presteered broadband beamforming based on worst-case performance optimization," in 5th IEEE SAM, Darmstadt, Germany, July 2008, pp. 340-344.

[12] W. Liu and S. Weiss, Wideband Beamforming - Concepts and Techniques, Wiley, 2010.

[13] M.R. Sayyah Jahromi and L.C. Godara, "Steering broadband beamforming without pre-steering," in IEEE/ACES Int. Conf. Wireless Comms \& Applied Comp. Electromag., Apr. 2005, pp. 987-990.

[14] J. Selva, "An efficient structure for the design of variable fractional delay filters based on the windowing method," IEEE Trans. SP, 56(8):3770-3775, Aug. 2008.

[15] M.A. Alrmah, S. Weiss, and J.G. McWhirter, "Implementation of accurate broadband steering vectors for broadband angle of arrival estimation," in IET Intelligent Signal Proc., London, UK, Dec. 2013.

[16] G.H. Golub and C.F. Van Loan, Matrix Computations, John Hopkins, 1996.

[17] S. Haykin, Adaptive Filter Theory, Prentice Hall, 1991.

[18] J.G. McWhirter, P.D. Baxter, T. Cooper, S. Redif, and J. Foster, "An EVD Algorithm for Para-Hermitian Polynomial Matrices," IEEE Trans. SP, 55(5):2158-2169, May 2007.

[19] J. Corr, K. Thompson, S. Weiss, I.K. Proudler, and J.G. McWhirter, "Row-shift corrected truncation of paraunitary matrices for PEVD algorithms," submitted to EUSIPCO, Nice, France, September 2015.

[20] S. Redif, S. Weiss, and J.G. McWhirter, "Sequential matrix diagonalization algorithms for polynomial EVD of parahermitian matrices," IEEE Trans. SP, 63(1):81-89, Jan. 2015. 